\title{
Characterization of Nano-Scale Defects in Pulsed Laser Deposited (PLD) Thin Films of Li3x Nd(2/3-x)(1/3-2x)TiO3 (NLTO) by Aberration Corrected HR-STEM Imaging and Dual-EELS
}

Robert Williams ${ }^{1}$, Nuria Bagues ${ }^{2}$, Elahe Farghadany ${ }^{3}$, Alp Sehirlioglu ${ }^{3}$ and David McComb ${ }^{4}$

${ }^{1}$ The Ohio State University, Hilliard, Ohio, United States, ${ }^{2}$ The Ohio State University, Columbus, Ohio, United States, ${ }^{3}$ Case Western Reserve Univeristy, Cleveland, Ohio, United States, ${ }^{4}$ Center for Electron Microscopy and Analysis/ Department of Material Science and Engineering, The Ohio State University, Columbus, Ohio, United States

Rechargeable batteries and chemical sensors have become a technological necessity with applications ranging from biomedical implants to cell phones to automobiles and beyond. With increased usage, reliability and safety concerns, along with demand for greater power density, the need for better material systems is growing. One possible solution is solid state battery technology which shows promise for faster charging, higher output voltage and longer life cycles. Solid state batteries are built using solid electrolytes, also known as fast ionic conductors, that allow the movement of ionic species through a rigid crystal lattice and if the activation energy for mobility is sufficiently low, the solid electrolyte can serve as both the separator and electrolyte for the battery. In the case of pulsed laser deposited(PLD) thin films, imperfections in growth parameters and variations in composition create a rich, defect landscape throughout the film; creating new, and perhaps, undesirable pathways. Characterizing the defects that arise and determining the effect that deposition parameters, such a Li content of target, cooling rate and film thickness play towards film growth is critical to understanding the ionic conductivity response and will be discussed in this work.

$(\mathrm{Li} 3, \mathrm{Nd}) \mathrm{TiO}_{3}(\mathrm{NLTO})$ is a material system that exhibits high ionic conductivity for Li and shows promise as a future solid-state electrolytic material. Bulk measurements indicate that grain boundaries decrease conductivity and crystal structure defects increase the Li conductivity of the material. For the case of PLD films, the desired goal was to produce epitaxial single crystals that created a preferred 2-D ionic pathway, however, achieving optimal growth parameters required optimizing processing windows for substrate temperature, background pressure, laser fluence, and pulse frequency. During parameter optimization, often a rich defect landscape formed that was a function of local chemistry, microstructure and crystal structure as shown in Fig. 1. The effect of defects on conductivity has been both positive and negative from bulk measurements, while the effects of defects on PLD films is unexplored.

For this work, thin films of NLTO have been deposited by PLD on (001) $\mathrm{SrTiO}_{3}$, with varying deposition parameters, in an attempt to produce epitaxial, single crystals that exhibit optimal ionic conductivity properties. PLD growths were monitored in-situ, by reflection high energy electron diffraction (RHEED), to observe layer growth and crystallinity; atomic force microscopy (AFM) was utilized to characterize the film's surface topography. Perhaps the most powerful tool for characterizing PLD solid-state thin films is aberration-corrected, monochromated scanning transmission electron microcopy ((S)TEM) which provided a sub-angstrom probe for imaging and an energy resolution of $<0.2 \mathrm{eV}$ for spectral analysis by electron energy loss spectroscopy (EELS). Direct observation and characterization of resultant PLD thin films was performed using a Thermo Scientific aberration corrected HAADF-STEM for imaging and dualEELS analysis to provide microstructural and chemical information. Various methods, such as integrated differential phase contrast (iDPC) and annular bright field (ABF), as well as EELS spectrum imaging were 
acquired at both room and cryo temperatures to observe the location of $\mathrm{Li}$, characterize defect structure and refine deposition parameters.

Special care was taken in preparing (001) cross - sectional TEM samples using a Helios Nanolab for bulk trenching and thinning. The foils were extracted using an Omniprobe 200 micromanipulator and mounted on a $\mathrm{Cu}$ grid in a flag orientation, final thinning and subsequent low $\mathrm{kV} \mathrm{Ar}{ }^{+}$milling at 900 and $500 \mathrm{eV}$ to remove amorphous ion damage and provide optimal surface quality for high resolution analysis.

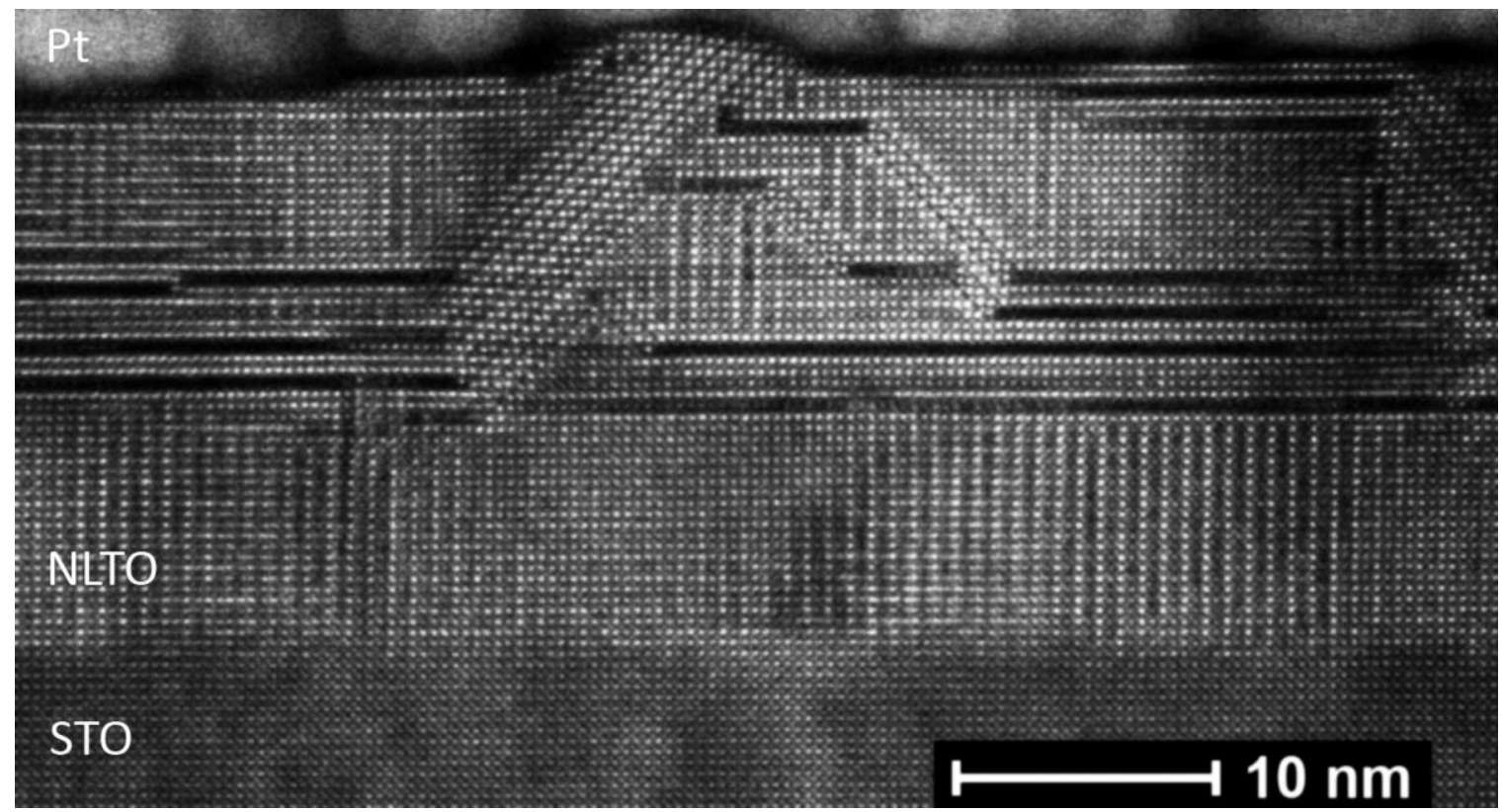

Figure 1. HAADF-STEM image of NLTO grown on (001) STO and capped with Pt from FIB extraction. Various defects can be observed in the NLTO film. The linear, black regions in the image are A-site deficiencies and would be ideal for Li conduction pathways. Note that the bottom half of the NLTO layer seems defect free, while having large intensity variations from column to column, above a critical thickness, A-site defects appear more frequently.

References

Dholabhai, P.P., et al, Nature Communications, 5:5043, 2014 . DOI:10.1038/ncomms6043

Stramere, S., et al, Chemical Materials, (15) 3974, 2003. 